\title{
The ADAM15 ectodomain is shed from secretory exosomes
}

\author{
Hee Doo Lee ${ }^{1}$, Yeon Hyang Kim², Bon-Hun $\mathrm{Koo}^{1}$ E Doo-Sik Kim ${ }^{1, *}$ \\ ${ }^{1}$ Department of Biochemistry, College of Life Science and Biotechnology, Yonsei University, Seoul 120-749, ${ }^{2}$ Department of Bioinformatics, \\ Korea Polytechnics, Nonsan 320-905, Korea
}

\begin{abstract}
We demonstrated previously that a disintegrin and metalloproteinase 15 (ADAM15) is released into the extracellular space as an exosomal component, and that ADAM15-rich exosomes have tumor suppressive functions. However, the suppressive mechanism of ADAM15-rich exosomes remains unclear. In this study, we show that the ADAM15 ectodomain is cleaved from released exosomes. This shedding process of the ADAM15 ectodomain was dramatically enhanced in conditioned ovarian cancer cell medium. Proteolytic cleavage was completely blocked by phenylmethylsulfonyl fluoride, indicating that a serine protease is responsible for exosomal ADAM15 shedding. Experimental evidence indicates that the ADAM15 ectodomain itself has comparable functions with those of ADAM15-rich exosomes, which effectively inhibit vitronectininduced cancer cell migration and activation of the MEK/extracellular regulated kinase signaling pathway. We present a tumor suppressive mechanism for ADAM15 exosomes and provide insight into the functional significance of exosomes that generate tumor-inhibitory factors. [BMB Reports 2015; 48(5): 277-282]
\end{abstract}

\section{INTRODUCTION}

Human a disintegrin and metalloproteinase 15 (ADAM15) is a type-I transmembrane glycoprotein composed of common domains, including propeptide, metalloproteinase, disintegrin-like, cysteine-rich, epidermal growth factor-like, transmembrane, and cytoplasmic domains $(1,2)$. The ADAM15 metalloproteinase domain has catalytic activity and is involved in ectodomain shedding of surface proteins, including cadherin, transforming growth factor- $\alpha$, and amphiregulin (3-6). Moreover, ADAM15 is the only ADAM member that contains an Arg-Gly-Asp motif in its disintegrin-like domain, which has tumor inhibitory activ-

*Corresponding author. Tel: +82-2-2123-2700; Fax: +82-2-3126027; E-mail: dskim@yonsei.ac.kr

http://dx.doi.org/10.5483/BMBRep.2015.48.5.161

Received 5 August 2014, Revised 24 August 2014, Accepted 4 September 2014

Keywords: ADAM15, Ectodomain shedding, Exosomes, MEK/ERK signaling, Tumor suppression ity $(7,8)$. However, the functional mechanism of ADAM15 remains unclear.

Exosomes are generated via the endocytic pathway and through budding in multivesicular bodies. Exosomes are secreted when the bodies fuse with the plasma membrane. Exosomes are characterized by their conserved size (80-100 $\mathrm{nm})$ and density $(1.11-1.18 \mathrm{~g} / \mathrm{ml})(9,10)$. The essential functions of exosomes, including intercellular communication and regulation of target cell gene expression have been revealed gradually $(9,10)$.

We demonstrated previously that ADAM15 is secreted as an exosomal component and that ADAM15-rich exosomes have tumor inhibitory activities (8). Here, we show that the ADAM15 ectodomain is shed from secretory exosomes. A functional investigation of the ADAM15 ectodomain revealed the underlying mechanism of ADAM15 exosome-mediated tumor suppression.

\section{RESULTS}

ADAM15 fragments are released into the extracellular space We demonstrated previously that mature ADAM15 is released via the exosome pathway, and that incorporation of ADAM15 into exosomes increases dramatically by phorbol 12-myristate 13-acetate (PMA)-mediated protein kinase $\mathrm{C}$ activation (8). We isolated ADAM15 exosomes from conditioned medium (CM) of ADAM15-transfected cells stimulated with PMA (Fig. 1A). Interestingly, the cytoplasmic fragment and full-length ADAM15 were detected in exosomes by immunoblotting with an antibody specific to the ADAM15 cytoplasmic domain (Fig. 1A). Glyceraldehyde-3-phosphate dehydrogenase, one of the most abundant exosomal proteins (11), was used as an internal control (Fig. 1A). We concentrated the CM of ADAM15-transfected HEK293F cells to identify the ADAM15 extracellular fragment. As shown in Fig. 1B, the ADAM15 extracellular fragment was successfully detected in the concentrated $C M$ with an antibody specific to the ADAM15 extracellular domain. Moreover, the ADAM15 cytoplasmic fragment was identified in concentrated CM by an antibody specific to the ADAM15 cytoplasmic domain, which contained exosomes (Fig. 1B).

\section{Ectodomain shedding of ADAM15 from exosomes}

Because ADAM15 cytoplasmic fragments were detected in exosomes, we focused on whether ADAM15 is shed from 
A

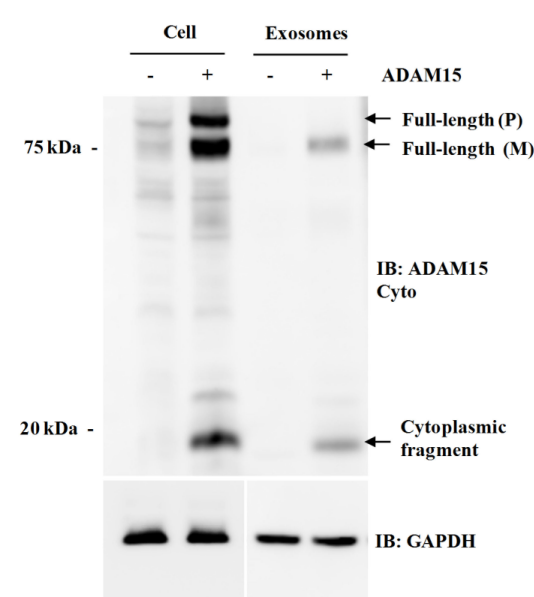

B

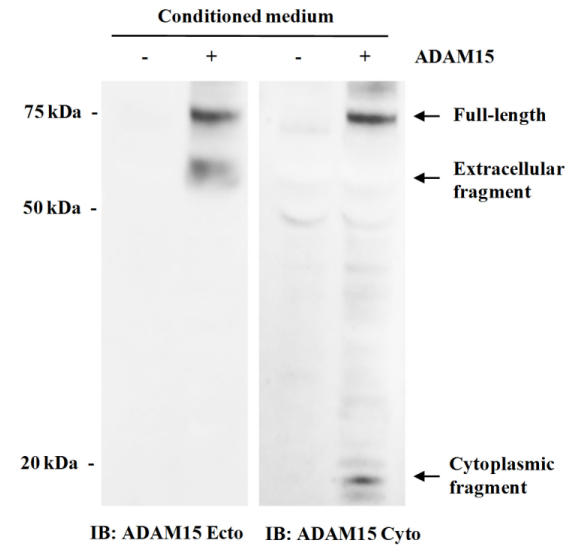

IB: ADAM15 Ecto IB: ADAM15 Cyto

Fig. 1. The a disintegrin and metalloproteinase 15 (ADAM15) ectodomain is detected in the extracellular compartment. (A) Immunoblotting (IB) analysis of ADAM15-transfected HEK293F cells and isolated ADAM15 exosomes with antibody against the ADAM15 cytoplasmic domain (ADAM15 Cyto). HEK293F cells were transiently transfected with an empty or the ADAM15 expression vector and incubated with $10 \mathrm{ng} / \mathrm{mL}$ phorbol 12-myristate 13-acetate (PMA) in serum-free medium for $24 \mathrm{~h}$. Cell lysates and isolated exosomes from conditioned medium $(\mathrm{CM})$ were analyzed by sodium dodecyl sulfate polyacrylamide gel electrophoresis (SDS-PAGE). $\mathrm{P}$ and $\mathrm{M}$ indicate the pro-form and mature-form of ADAM15, respectively. (B) Western blotting analysis of CM with antibody against the ADAM15extracellular (ADAM15 Ecto) or cytoplasmic domain (ADAM15 Cyto). ADAM15-transfected HEK293F cells were incubated with $5 \mathrm{ng} / \mathrm{ml} \mathrm{PMA}$ in serum-free medium for $24 \mathrm{~h}$. The CM was concentrated prior to SDS-PAGE.

A

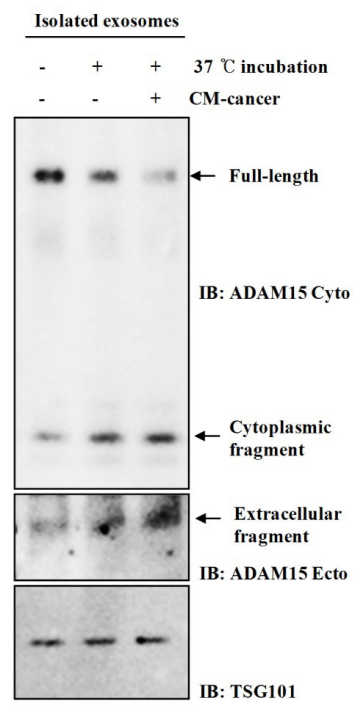

B

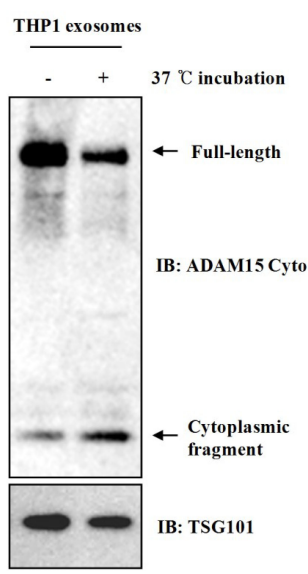

C

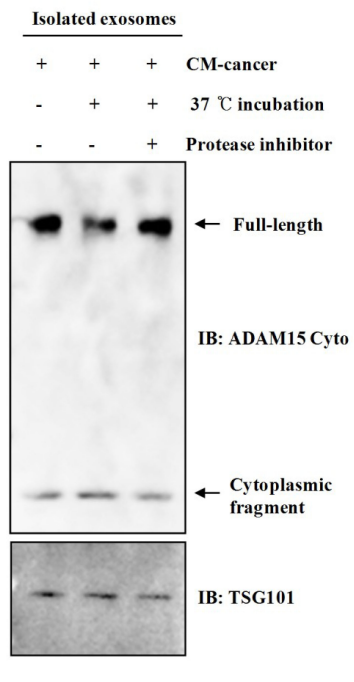

Fig. 2. Ectodomain shedding of a disintegrin and metalloproteinase 15 (ADAM15) from exosomes. (A) ADAM15-rich exosomes were isolated from ADAM15-transfected 293F cells stimulated with phorbol 12-myristate 13-acetate (PMA) for $24 \mathrm{~h}$. The purified exosomes were further incubated at $37^{\circ} \mathrm{C}$ for $10 \mathrm{~h}$ with or without conditioned medium from MDAH2774 ovarian cancer cells (CM-cancer). The samples were analyzed by immunoblotting with the indicated antibodies. (B) THP1 cells were induced to undergo monocytic differentiation with $20 \mathrm{ng} / \mathrm{ml} \mathrm{PMA}$ for $24 \mathrm{~h}$, and exosomes were isolated from CM of differentiated THP1 cells. Purified exosomes were further incubated as described above. The samples were analyzed by immunoblotting with the indicated antibodies. (C) Exosomes were purified and incubated with universal protease inhibitor (PI) as described above. The samples were analyzed by immunoblotting with the indicated antibodies. 
exosomes. To test this, purified ADAM15-rich exosomes were incubated at $37^{\circ} \mathrm{C}$. As shown in Fig. $2 \mathrm{~A}$, the level of full-length ADAM15 was reduced in exosomes, corresponding with an increase in the cytoplasmic fragment level following an incubation at $37^{\circ} \mathrm{C}$. We also observed that the ADAM15 extracellular fragment level increased in the incubated exosomes, indicating shedding of ADAM15 from exosomes (Fig. 2A). In contrast, the protein level of TSG101, an exosomal marker protein present in the internal exosome space (12), did not change significantly after the $37^{\circ} \mathrm{C}$ incubation. As the ADAM15-rich exosomes have tumor inhibitory activity (8), we explored whether cancer cells affect the shedding activity of ADAM15 from exosomes. Notably, ADAM15 shedding increased dramatically when the purified exosomes were further incubated with CM from MDAH2774 cancer cells (Fig. 2A), suggesting that cancer cell-derived factors may regulate shedding of ADAM15 from exosomes.

We showed previously that ADAM15 exosomes are actively released from differentiated THP1 cells (8). ADAM15 exosomes were isolated from differentiated THP1 cells and further incubated at $37^{\circ} \mathrm{C}$ with $\mathrm{CM}$ from MDAH2774 cancer cells to investigate ADAM15 shedding activity in exosomes derived from non-transfected (native) cells. As shown in Fig. 2B, ADAM15 shedding occurred in the THP1-derived exosomes, suggesting the physiological phenomena of ADAM15 shedding in innate immune cells.

Many studies have reported that ectodomain shedding of transmembrane proteins is caused by serine or metalloproteases (13-17). We found that ADAM15 shedding was blocked completely by broad-spectrum protease inhibitors (Roche 11873580001) (Fig. 2C). To explore this phenomenon more specifically, we used phenylmethylsulfonyl fluoride (PMSF), a serine protease inhibitor, and ethylenediaminetetraacetic acid (EDTA), a metalloprotease inhibitor. As shown in Fig. 3A and $B$, shedding of ADAM15 from isolated exosomes was successfully blocked by PMSF, indicating that a serine protease participates in shedding of ADAM15 from exosomes.

\section{The ADAM15 ectodomain suppresses vitronectin-induced cell migration and activation of MEK/extracellular regulated kinase (ERK) signaling}

ADAM15-rich exosomes suppress cancer cell migration by inhibiting integrin $\alpha v \beta 3$ (8). As MDAH2774 cancer cell-released factors significantly induce ADAM15 ectodomain shedding from exosomes, we explored the effect of the ADAM15 ectodomain on cancer cell migration. As shown in Fig. 4A, vitronectin dramatically induced Transwell migration of MDAH2774 ovarian cancer cells, and vitronectin-induced cell migration was effectively suppressed by ADAM15-rich exosomes, as well as by the recombinant ADAM15 ectodomain.

Vitronectin promotes cell migration by integrin-mediated activation of the MEK/ERK signaling pathway (18). We confirmed that MEK and ERK phosphorylation increased significantly in response to vitronectin (Fig. 4B). Notably, both types of phosphorylation were suppressed by the ADAM15 ectodomain, as well as by ADAM15-rich exosomes, indicating the inhibitory effect of released ADAM15 on the vitronectin-induced signaling pathway.

\section{DISCUSSION}

Our data lead to several important findings. First, the ADAM15 ectodomain is shed from secretory exosomes. Second, shedding of the ADAM15 ectodomain was induced by cancer
A

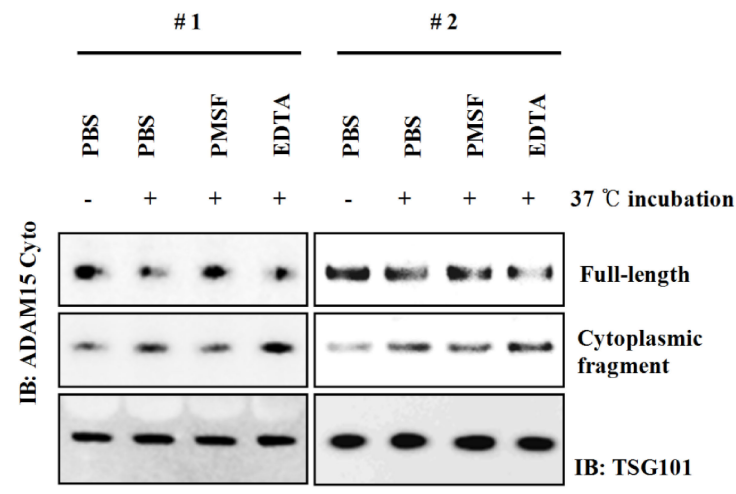

B

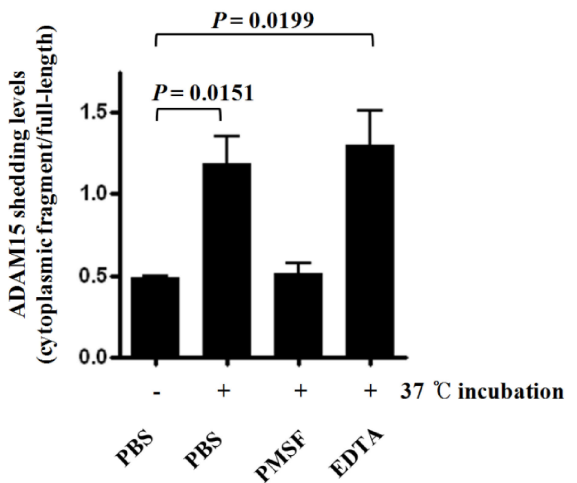

Fig. 3. Effect of specific protease inhibitor on a disintegrin and metalloproteinase 15 (ADAM15) shedding. (A, B) Purified exosomes were incubated in the presence or absence of $1 \mathrm{mM}$ phenylmethylsulfonyl fluoride (PMSF) or ethylenediaminetetraacetic acid (EDTA). The samples were analyzed by immunoblotting with the indicated antibodies. \#1 and \#2 indicate sets of independent experiments (A). Tight panel represents the mean \pm standard deviation of three independent experiments (B). ADAM15 shedding levels are expressed as signal intensity ratios (cytoplasmic fragment/full-length). P-values were calculated using the unpaired Student's $t$-test. 
A
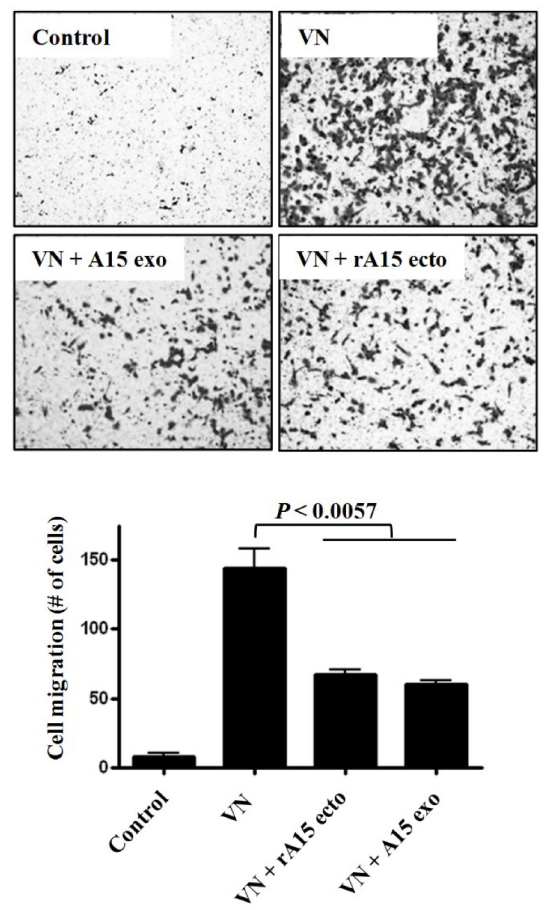

B

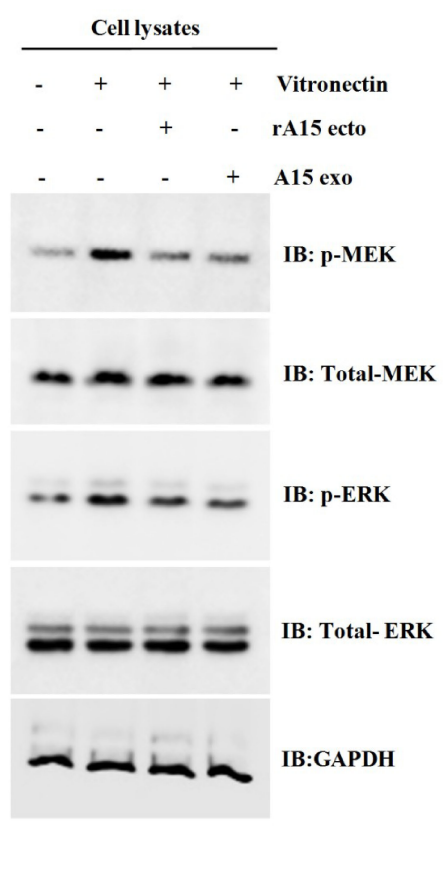

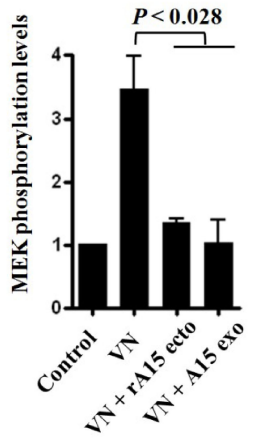

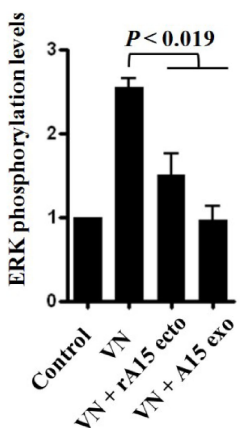

Fig. 4. A disintegrin and metalloproteinase 15 (ADAM15) ectodomain inhibits vitronectin-induced cell migration and MEK/extracellular regulated kinase (ERK) activation. (A) Effect of the ADAM15 ectodomain on vitronectin-induced cell migration. MDAH2774 cells were placed in an $8-\mu \mathrm{m}$ pore-sized Transwell insert, and migration of the cells toward vitronectin $(1.25 \mu \mathrm{g} / \mathrm{ml})$ was analyzed in the presence of the recombinant ADAM15 ectodomain (rA15 ecto: $0.15 \mu \mathrm{M}$ ) or ADAM15-rich exosomes (A15 exo: $2 \mu \mathrm{g}$ ). After a $16 \mathrm{~h}$ incubation, the number of migrated cells in each field was determined by light microscopy $(20 \times$ original magnification) and Image software. Lower panel represents the mean \pm standard deviation (SD) of three independent experiments. (B) Effect of the ADAM15 ectodomain on vitronectin-induced activation of MEK/ERK signaling. MDAH2774 cells were cultured on vitronectin-coated plates (1.25 $\mu \mathrm{g} / \mathrm{ml})$ in the presence of the ADAM15 ectodomain (rA15 ecto: $0.15 \mu \mathrm{M}$ ) or ADAM15-rich exosomes (A15 exo: $2 \mu \mathrm{g}$ ) for $1 \mathrm{~h}$. MEK or ERK phosphorylation was analyzed by immunoblotting (IB) with the indicated antibodies. Right panels represent mean \pm SD of three independent experiments. Phosphorylation levels are expressed as signal intensity (fold-change relative to control). P-values were calculated using the unpaired Student's t-test.

cell-released factors, and was associated with serine protease activity, not metalloprotease activity. Finally, the ADAM15 ectodomain suppressed cancer cell migration and activation of the MEK/ERK signaling pathway.

The most interesting finding is that the ADAM15 ectodomain was shed from secretory exosomes. It has been suggested that exosomes are released as a platform for ectodomain shedding of transmembrane proteins (19). For example, the L-1 protein ectodomain is shed from exosomes and affects target cell migration $(20,21)$. It is thought that the release of exosomes via the endocytic pathway increases both protease activity and the delivery of secretory molecules to target cells. Thus, we hypothesized that ADAM15 is an important functional factor released from exosomes.

Surprisingly, ADAM15 shedding is completely blocked by serine protease-specific inhibitors. Serine proteases participate in diverse shedding processes of transmembrane proteins, in- cluding E-cadherin, L-1, gAce, syndecan-1, and syndecan-4 (14-17). Our results indicate that serine proteases play an essential role in exosome-mediated protein release.

Shedding activity effectively increases by factors secreted from cancer cells. Although we could not clarify whether the secreted factors included a serine protease or an inducer of serine protease activity, this phenomenon indicates that cancer cells modulate exosome function through their secretory factors. Therefore, further study is necessary to elucidate the underlying mechanism of cancer cell-induced ADAM15 shedding.

Our earlier study demonstrated that ADAM15 exosomes exhibit tumor suppressive functions by blocking integrin $\alpha v \beta 3$ (8). However, the underlying mechanism is not fully understood. The question asked in that study was how ADAM15 packed in exosomes successfully covered dispersed integrin $\alpha v \beta 3$ on the cell surface. Here, we suggest that the ectodomain shedding of ADAM15 is crucial for ADAM15 exosome- 
mediated tumor suppression. Hypothetically, ADAM15 is efficiently shed from exosomes near cancer cells, and the ADAM15 ectodomain released effectively inhibits cancer progression by blocking the integrin-mediated MEK/ERK signaling pathway.

We found here that extracellular fragments of ADAM15 are released via exosomes, and demonstrated the biological function of the ADAM15 ectodomain as a tumor inhibitor. This finding provides insight into how ADAM15 exosomes participate in tumor suppression.

\section{MATERIALS AND METHODS}

\section{Materials}

Mouse monoclonal antibodies specific to the ADAM15 extracellular domain and pERK were purchased from Santa Cruz Biotechnology (Santa Cruz, CA, USA). Rabbit polyclonal anti-ADAM15 cytoplasmic domain antibody (AB19036) and metalloproteinase domain antibody were purchased from Chemicon (Temecula, CA, USA) and Abcam (Cambridge, U K), respectively. PMA (P1585) and the recombinant human ADAM15 ectodomain (WBC027) were purchased from SigmaAldrich (St. Louis, MO, USA) and R\&D Systems (Minneapolis, $\mathrm{MN}, \mathrm{USA})$, respectively.

\section{Cell culture}

MDAH2774 (human ovarian adenocarcinoma, ATCC no. CRL10303) and HEK293F (human embryonic kidney) cells were maintained in Dulbecco's modified Eagle's medium containing $10 \%$ fetal bovine serum (FBS). MDAH2774 cells were starved with serum-free medium for $2 \mathrm{~h}$ before the experiments. THP1 (human monocytes, ATCC no. TIB-202) cells were maintained in RPMI 1640 medium containing 10\% FBS.

\section{Expression plasmid, site-directed mutagenesis, and transfection}

The expression plasmid containing full-length ADAM15 (accession no. NM_003815.3) was described previously (8). A catalytically inactive mutant was generated by site-directed mutagenesis (Intron Biotechnology, Daejeon, Korea). The expression plasmid was transfected according to the manufacturer's recommendations (Invitrogen, Carlsbad, CA, USA).

\section{Exosome isolation}

Exosomes were isolated as described previously (12). Cells were incubated with or without PMA in serum-free medium in 100-mm culture dishes for $24 \mathrm{~h}$. The conditioned medium $(\mathrm{CM})$ was sequentially centrifuged at $300 \times \mathrm{g}$ for $10 \mathrm{~min}$, and $1200 \times \mathrm{g}$ for $20 \mathrm{~min}$ to remove cellular debris. The resulting supernatants were passed through a $0.22-\mu \mathrm{m}$-pore filter (Millipore, Billerica, MA, USA) to remove shed vesicles from microvesicles (22), and was then subjected to ultracentrifugation at $100,000 \times g$ for $1 \mathrm{~h}$ in a Beckman SW 55 Ti rotor. The pellets were resuspended in 50-100 $\mu$ l phosphate-buffered saline (PBS) and stored at $-80^{\circ} \mathrm{C}$. Protein concentration was measured with a modified Bradford assay (Bio-Rad Laboratories, Hercules, CA, USA).

\section{Exosomal ADAM15 shedding assay}

Exosomes were isolated from CM of ADAM15-transfected HEK293F cells stimulated with PMA for $24 \mathrm{~h}$. The isolated exosomes were subjected to further incubation at $37^{\circ} \mathrm{C}$ for 10 h in PBS with or without CM of MDAH2774 cancer cells. Samples were lysed and analyzed by sodium dodecyl sulfate-polyacrylamide gel electrophoresis (SDS-PAGE).

\section{Cell migration assay}

Cell migration assays were performed using Transwell inserts with 6.5-mm diameter polycarbonate 8 - $\mu \mathrm{m}$ microporous membranes (Costar; Cambridge, MA, USA). Cells $\left(3 \times 10^{4}\right)$ were placed in the inner chamber, and $0.6 \mathrm{~mL}$ of serum-free medium was added to the outer chamber with or without vitronectin $(1.25 \mu \mathrm{g})$. After a $16 \mathrm{~h}$ incubation, the migrated cells were stained with hematoxylin. The number of migrated cells was determined by light microscopy and ImageJ software.

\section{Statistical analysis}

All experiments were repeated at least three times, yielding consistent results. Data are presented as mean \pm standard deviation (SD) of $\mathrm{n}$ independent experiments. The statistical analysis was performed using unpaired Student's t-tests. A P-value $<0.05$ was considered significant.

\section{ACKNOWLEDGEMENTS}

This study was supported by the Korean National Research Foundation (NRF) Grant 2012M3A9A9055078.

\section{REFERENCES}

1. Wolfsberg TG, Primakoff $P$, Myles DG and White JM (1995) ADAM, a novel family of membrane proteins containing A Disintegrin And Metalloprotease domain: multipotential functions in cell-cell and cell-matrix interactions. J Cell Biol 131, 275-278

2. Zhang XP, Kamata T, Yokoyama K, Puzon-McLaughlin W and Takada $Y$ (1998) Specific interaction of the recombinant disintegrin-like domain of MDC-15 (metargidin, ADAM-15) with integrin alphavbeta3. J Biol Chem 273, 7345-7350

3. Najy AJ, Day KC and Day ML (2008) The ectodomain shedding of E-cadherin by ADAM15 supports ErbB receptor activation. J Biol Chem 283, 18393-18401

4. Schafer B, Gschwind A and Ullrich A (2004) Multiple G-protein-coupled receptor signals converge on the epidermal growth factor receptor to promote migration and invasion. Oncogene 23, 991-999

5. Schafer B, Marg B, Gschwind A and Ullrich A (2004) Distinct ADAM metalloproteinases regulate $G$ protein-coupled receptor-induced cell proliferation and survival. J Biol Chem 
279, 47929-47938

6. Najy AJ, Day KC and Day ML (2008) ADAM15 supports prostate cancer metastasis by modulating tumor cell-endothelial cell interaction. Cancer Res 68, 1092-1099

7. Hou Y, Chu M, Du FF et al (2013) Recombinant disintegrin domain of ADAM15 inhibits the proliferation and migration of Bel-7402 cells. Biochem Biophys Res Commun $435,640-645$

8. Lee HD, Koo BH, Kim YH, Jeon $\mathrm{OH}$ and Kim DS (2012) Exosome release of ADAM15 and the functional implications of human macrophage-derived ADAM15 exosomes. FASEB J 26, 3084-3095

9. Cocucci E, Racchetti G and Meldolesi J (2009) Shedding microvesicles: artefacts no more. Trends Cell Biol 19, 43-51

10. Thery C, Ostrowski M and Segura E (2009) Membrane vesicles as conveyors of immune responses. Nat Rev Immunol 9, 581-593

11. Mathivanan S and Simpson RJ (2009) ExoCarta: A compendium of exosomal proteins and RNA. Proteomics 9, 4997-5000

12. Lee HD, Kim YH and Kim DS (2013) Exosomes derived from human macrophages suppress endothelial cell migration by controlling integrin trafficking. Eur J Immunol 44, 1156-1169

13. Weber S and Saftig P (2012) Ectodomain shedding and ADAMs in development. Development 139, 3693-3709

14. Nayeem N, Silletti S, Yang X et al (1999) A potential role for the plasmin(ogen) system in the posttranslational cleavage of the neural cell adhesion molecule L1. J Cell Sci 112 (Pt 24), 4739-4749
15. Thimon V, Metayer S, Belghazi M, Dacheux F, Dacheux JL and Gatti JL (2005) Shedding of the germinal angiotensin I-converting enzyme (gACE) involves a serine protease and is activated by epididymal fluid. Biol Reprod 73, 881-890

16. Subramanian SV, Fitzgerald ML and Bernfield M (1997) Regulated shedding of syndecan- 1 and -4 ectodomains by thrombin and growth factor receptor activation. J Biol Chem 272, 14713-14720

17. Klucky B, Mueller R, Vogt I et al (2007) Kallikrein 6 induces E-cadherin shedding and promotes cell proliferation, migration, and invasion. Cancer Res 67, 8198- 8206

18. Salasznyk RM, Klees RF, Hughlock MK and Plopper GE (2004) ERK signaling pathways regulate the osteogenic differentiation of human mesenchymal stem cells on collagen I and vitronectin. Cell Commun Adhes 11, 137-153

19. Keller S, Sanderson MP, Stoeck A and Altevogt P (2006) Exosomes: from biogenesis and secretion to biological function. Immunology Lett 107, 102-108

20. Stoeck A, Keller S, Riedle S et al (2006) A role for exosomes in the constitutive and stimulus-induced ectodomain cleavage of L1 and CD44. Biochem J 393, 609-618

21. Gutwein P, Stoeck A, Riedle S et al (2005) Cleavage of L1 in exosomes and apoptotic membrane vesicles released from ovarian carcinoma cells. Clin Cancer Res 11, 24922501

22. Valadi H, Ekstrom K, Bossios A, Sjostrand M, Lee JJ and Lotvall JO (2007) Exosome-mediated transfer of mRNAs and microRNAs is a novel mechanism of genetic exchange between cells. Nat Cell Biol 9, 654-65 